\title{
An Exploratory Data Analytics Platform for Factories of Future
}

\author{
Engin Zeydan \\ Centre Technologic de Telecomunicacions de Catalunya, \\ Castelldefels, Barcelona, Spain, 08860. \\ engin.zeydan@cttc.cat
}

\author{
Omer Dedeoglu \\ Broadband Investment and Planning Department, \\ Türk Telekom, Istanbul, Turkey, 34889. \\ omer.dedeoglu@tuktelekom.com.tr
}

\begin{abstract}
Factories of Future (FoF) is an emerging vertical sector towards 5G network evolution. Fine-grained monitoring the network performance of FoF environment can help to extract insight on the quality-of-service $(\mathrm{QoS})$ of a given industrial service provided by next generation cellular technologies. However, one of the main solutins that mobile network operators (MNOs) are investing today is on data evaluation tools that can be integrated withing their network infrastructure. In this paper using the open-source analytics tools, we analyze the industrial network traffic characteristics behaviour of an operational cellular network of a MNO. Our results finds out the relationship between various key performance indicators (KPIs) and extract insights on the performance and operational aspects of a factory environment using the cellular networks data with ElasticSearch stack's data analytics capabilities.
\end{abstract}

Index Terms-factories of future, vertical sectors, cellular data, data analytics, ElasticSearch.

\section{INTRODUCTION}

"4th Industrial Revolution" has provided a drastic burst in technology and digital applications and changed the way traditional manufacturing depends on. Smart and connected factories lie at the core of Industry 4.0. Smart factories are uniting the computing power of servers with the recent developments on sensor and machinery technologies to provide a fully connected network. Simultaneously, wireless industrial Internet of things (IoT) deployment is also driven by strong industrial demand [1]. Therefore, Industry 4.0 is seen as one of the prominent example where dramatic changes of $5 \mathrm{G}$ can be observed. Together with 5G's capacity and speed advancements, more sensor devices and assets will be connected with a single network while boosting the visibility for Mobile Network Operators (MNOs) and equipment vendors.

$5 \mathrm{G}$ will bring fast, reliable, massive and low latency communication capabilities while handling influx of connected everything thanks to scalable and reliable connections between massive devices. Together with the dynamic and flexible design of 5G networks, smart Factories of Future (FoF) will be able bring new innovative and disruptive technologies into the factory environment and adjust the network based on their requirements. Better Augmented Reality (AR)/Virtual Reality (VR) connections provided by manufacturing companies and MNOs thanks to large bandwidth opportunities, improved communication between Automatic Guided Vehicles (AGVs)-

978-1-7281-1244-2/19/\$31.00 (C)2019 IEEE server thanks to Multi-acccess Edge Computing (MEC) capabilities, untethered robots with seamless wireless coverage, remote control of most of the production line machines without touching thanks to Ultra reliable and low latency communication (URLLC), the flexibility to work with factory equipment at any place on factory floor and visualize machine and network related issues can all be possible with the capabilities of $5 \mathrm{G}$ networks.

Together with 5G's massive IoT connectivity ecosystem, MNOs need to rely on data analytics to make smart, quick and reliable decisions. Data processing, analytics and visualization are going to be a key features of the factories of future since large amounts of data need to be processed in real-time and efficient decision making is required. Recent technological advancements in data processing pipelines have also provided advanced support for data collection, transfer and enriching analysis [2], [3]. Many data analytics and processing tools have emerged in recent years [4], [5]. Most of them are also optimized for different use cases and dataset characteristics, e.g. the works in [6], [7] concentrate on streaming data analytics, the works in [8], [9] are used for batch data processing purposes. Key challenges between Industry 4-0 and High Performance Computing (HPC) to accommodate high volume and computational demands is presented in [10]. Developing industrial automation systems to deliver next generation smart factory services are studied in H2020 project called DISTRUPT ${ }^{1}$. Another H2020 Clear5G project investigates the key enablers of Machine Type Communications (MTC) traffic for $5 \mathrm{G}$ in $\mathrm{FoF}^{2}$.

Currently, cellular data analytics inside factory environment is used in small amount to monitor changes in important Key Performance Indicators (KPIs) at the network level. Eventually, data analytics could also be tailored to give insights to third party application developers, factory owners, equipment vendors and users in addition to MNOs if appropriate data analytics pipeline is built. For example, when there exists large amount of data that can exceed the processing capacity of the network, the network quality diminishes. As a result,

\footnotetext{
${ }^{1}$ Decentralised architectures for optimised operations via virtualised processes and manufacturing ecosystem collaboration (DISRUPT) project. http://www.disrupt-project.eu/

${ }^{2}$ Converged wireless access for reliable 5G MTC for factories of the future (Clear5G) project. http://clear5g.eu/
} 
data analytics and visualization platforms can be tuned to provide short to medium term feedback to the service provider or infrastructure owner when the data rate in a factory environment changes dynamically depending on the time of the day/month as well as the location of the cell. Moreover, data analytics will not just be used to give insights to these main actors and stakeholders, but it could also be used to predict important issues in the production lines, cellular networks and to assess new business opportunities. For example, major benefits can arise for equipment vendors if they can predict the malfunctioning of their products in near real-time depending on the real-time traffic load. These insights can increase performance, improve operational efficiency as well as ensure a safety environment for factories.

In this paper different than above related works, we perform a Exploratory Data Analytics (EDA) on the historical cellular network data of Base Stations (BSs) collected from the internal factory environment using open-source analytics tool ElasticSearch. In order to perform EDA, first we give our data analytics pipeline and each utilized component' definitions for the open-source analytics software used throughout our analysis. Second, we define some of the most relevant KPIs that may be valuable from network performance standpoint of the internal factory environment. Later, we discuss about our exploratory analysis approach. Our results yield interesting insights to MNOs as well as to factory owners that can be used for future network planning and capacity adjustments. The rest of the paper is organized as follows: In Section II, we provide the data analytics architecture for FoF and define the utilized KPIs and the dataset characteristics. In Section III, we give EDA results using the ElasticSearch data analysis architecture. Finally, in Section IV we provide the conclusions and future works of the paper.

\section{A Data AnAlytics Architecture FOR FoF}

In fully connected and automated smart factories, there will be heterogeneous sources of data which need to be aggregated at a central data visualization and analytics platform as given in Fig. 3. Therefore, leveraging from smart factory data requires data processing, analysis and insight extraction processes. Integrating those cutting-edge data analytics platform in a smart factory environment can ensure to quickly adapt to dynamic network conditions and user/device network behaviour. This will help agile and faster decisions.

Five main layers and corresponding components of the proposed data analytics architecture are shown in Fig. 2. These components are MNOs factory performance data source inside Data Source Layer, ElasticSearch Stack's Logstash placed inside Data Aggregation Layer, ElasticSearch data analysis tool placed inside Data Processing and Storage Layer, Map Visualization, Pandas and Kibana are connected to ElasticSearch inside Data Analytics and Visualization Layer and user is analyzing the results in Presentation Layer.

Using data analytics platform, cellular network data of factory cellular network is first collected inside the Elastic-

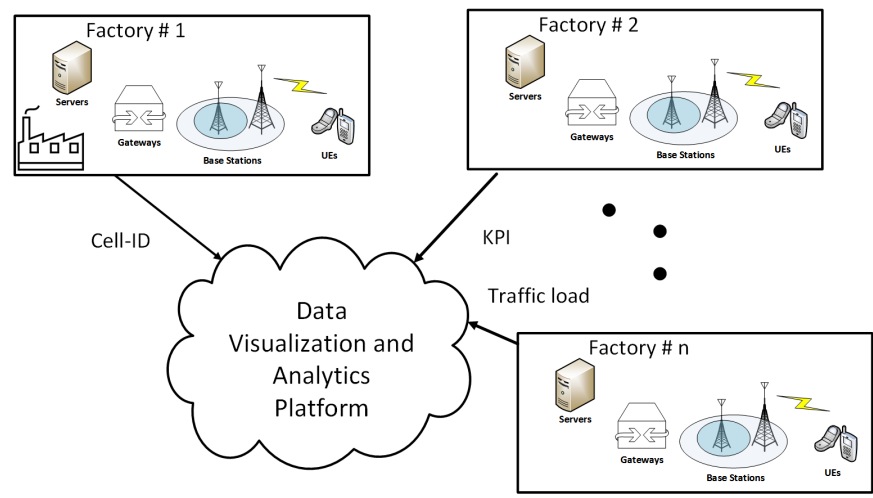

Fig. 1: Data collection process from different factories.

Search stack. ElasticSearch Stack is an open source search and analytics tool that can provide searching, analyzing and visualizing the MNOs' network performance data [11]. Dataset marked as step-1 in Fig. 2 is the cellular network data that is collected from indoor and outdoor antennas location in different factories that are serviced by MNO. After that in step-2, the data are pre-processed and data cleansing is performed. In this step, all the related fields for the considered factories are transformed e.g. into the CSV format or file system market as step-3. In Data Aggregation Layer, LogStash marked as step-4 provides server side data processing pipeline in order to ingest from multiple sources simultaneously [12]. Logstash is used to listen and transform as well load the data into ElasticSearch's output data format. Later, the data is stored using ElasticSearch for further analysis. ElasticSearch marked as step-5 enables query operation over stored structured and unstructured data in Data Processing and Storage Layer. Third party visualization tools can also be embedded into Elastic Stack as marked by step-7 map visualization. Data Analytics and Visualization Layer is used to spot infrastructure problems or business issues based on previously modeled behavior of the factory network data, e.g. trends, periodicity so that some notifications and actions can be taken based on the analysis results. Identification of issues, root cause analysis and reduction of false positives can also be some of the analysis that can be performed. Pandas marked as step-8, is used as a data analysis tool utilizing data-centric Pandas packages. Elastic Kibana [13] enables data visualization, interactive exploration and dashboard presentation of the network performance data of MNOs in Data Analytics and Visualization Layer. Kibana marked in step- 6 is an analytics and visualization platform and is used for easy tracking and visualization purposes of historical data by the interacting user in step-9.

Monitored KPIs and Dataset: There are various technical KPIs that are constantly monitored by MNOs. Moreover, those KPIs can also vary depending on the area domain. Some specific domains include Radio Access Network (RAN), Core and Transport networks, customer experience tools, network equipments in IT and operational/process tools. In our dataset, 


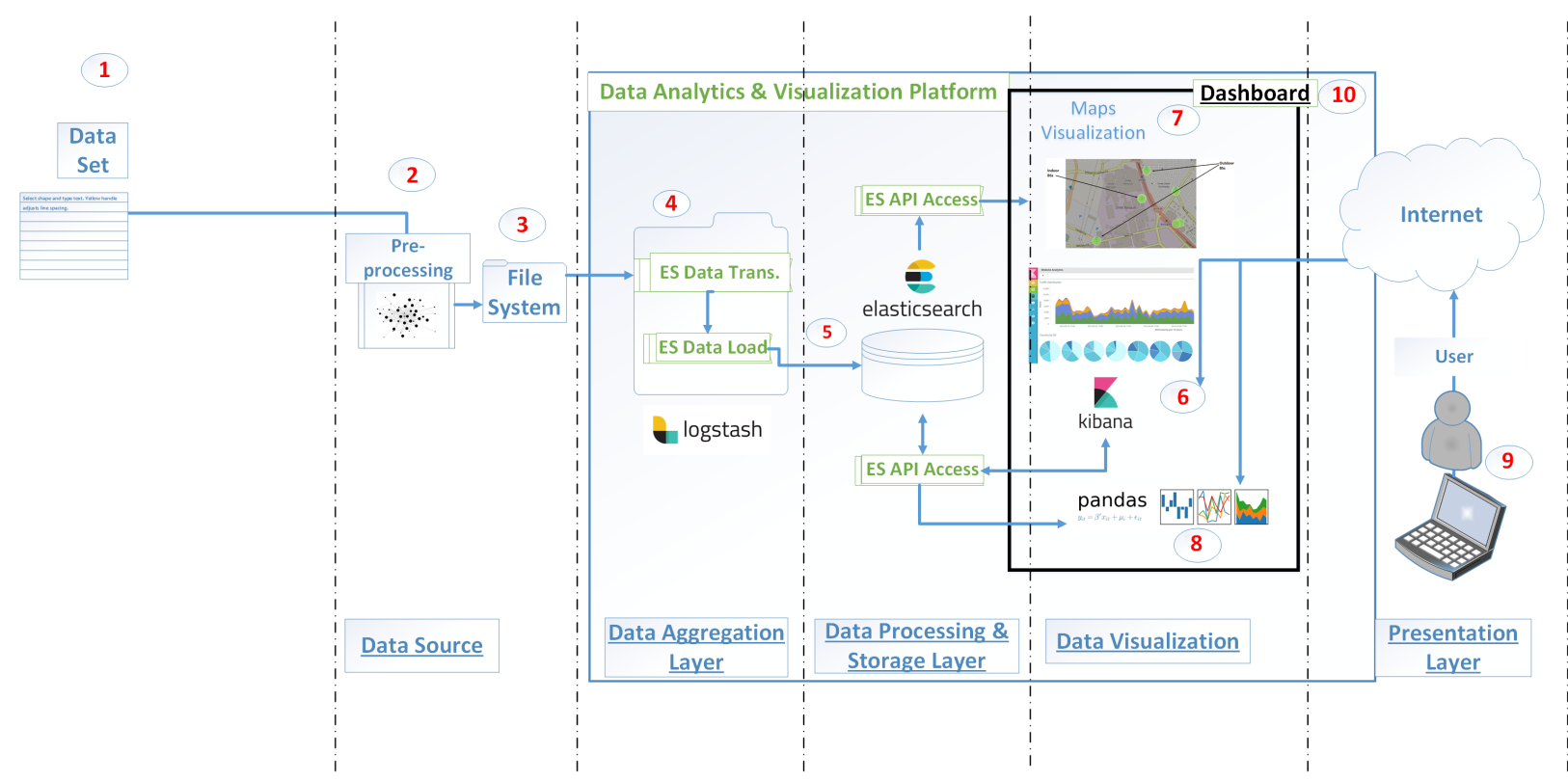

Fig. 2: General architecture of data analytics and visualization platform.

we follow the following fields in packet switched (PS) domain:

- Network Quality Index (NQI): The formula for NQI is defined as

$$
N Q I=A c c \times \operatorname{Ret} / 100
$$

- Retainability (Ret) : The formula for retainability is defined as

$$
\text { Ret }=100-R A B_{D R}
$$

- Accessibility $(A c c)$ : The formula for accessibility is defined as

$$
A c c=R R C_{S S R} \times R A B_{S S R} / 100
$$

- Traffic Load per hour: This field gives the amount of traffic consumed by a given Cell-ID in a given

where Radio Resource Control (RRC) Setup Success Rate is abbreviated as $R R C_{S S R}$, RRC Drop Rate as $R R C_{D R}$, radio access bearer (RAB) Setup Success Rate as $R A B_{S S R}$, RAB Drop Rate as $R A B_{D R}$. Additionally, Date Time, Site ID, Cell ID and City-District pairs are also available.

\section{EVAluations USING EXPLORATORY DATA ANALYSiS}

Our analysis results in this subsection is focusing on real network data traffic in one of the automobile factory in Turkey. ${ }^{3}$ In the considered automobile factory, there are outdoor and indoor antennas. The considered factory is divided into two main areas based on operational units: First area is main building and the second area is production building. Nine cells are present inside both main and production buildings. Cell-IDs inside

\footnotetext{
${ }^{3}$ Although other factories data can also be analyzed using the proposed data analytics platform.
}

main building are WBR17110, WBR17111, WBR17112, WBR17113, WBR17115, WBR17116, WBR17117, WBR17118 and WBR17119. Cell-IDs inside production buildings are: WBR17130, WBR17131, WBR17132, WBR17133, WBR17135, WBR17136, WBR17137, WBR17138, WBR17139. For below observations, we only focus on $3 \mathrm{G}$ traffic (excluding $2 \mathrm{G}$ traffic). Fig. 3 on the other hand shows the dashboard visualization of the Kibana for visualizing the factory data. A summary of the statistical distributions of the observed test data are given in Table I. The collected data is from $3 \mathrm{G}$ cellular technology ${ }^{4}$ The observation duration is between 04 July 2017 and 03 October 2017.

Fig. 4 shows the total UL and DL traffic over all the cells inside the factory. We can observe that there exists a periodicity within the observed dataset, e.g. small amount of traffic is generated on Sundays. We can also observe that traffic characteristics of UL and DL traffic are same. In the rest of the analysis, we focus on UL traffic without loss of generality.

Fig. 5 shows the factory cell Uplink (UL) median values of each weekday-hour time zone ${ }^{5}$ for UL heatmap over the observed period. We can observe that high activity occurs during working hours between 7 am to $5 \mathrm{pm}$ from Monday to Friday. Obviously, weekday traffic is more active than weekend traffic, Saturdays traffic concentrates mostly around $11 \mathrm{am}$ and almost no traffic is generated on Sundays. The results indicate that the automobile factory is mostly not active on Sundays and some small activity occurs on Saturdays. Interestingly, we can also observe that during $11 \mathrm{pm}$,

\footnotetext{
${ }^{4} 4 \mathrm{G}$ factory data was not available during the time of data collection inside the considered factories.

${ }^{5}$ each time zone is represented by weekday and hour
} 


\begin{tabular}{|l|l|l|l|}
\hline \# of measurements & 65,535 & \# of unique site-cell ID pairs & 43 \\
\hline \# of factories & 3 & $N Q I$ (average) & 98.898 \\
\hline Ret (average) & 99.714 & $A c c$ (average) & 99.179 \\
\hline DL (average, kbps) & $147,906.402$ & UL (average, kbps) & $21,009.467$ \\
\hline$R R C_{S S R}$ (average, \%) & 99.596 & $R R C_{D R}$ (average, \%) & 0.404 \\
\hline$R A B_{S S R}$ (average, \%) & 99.574 & $R A B_{D R}$ (average, \%) & 0.286 \\
\hline Obser. Duration & 91 days & Total \# of city-district pairs & 6 \\
\hline
\end{tabular}

TABLE I: Summary statistics of the whole network factory data inside ElasticSearch.
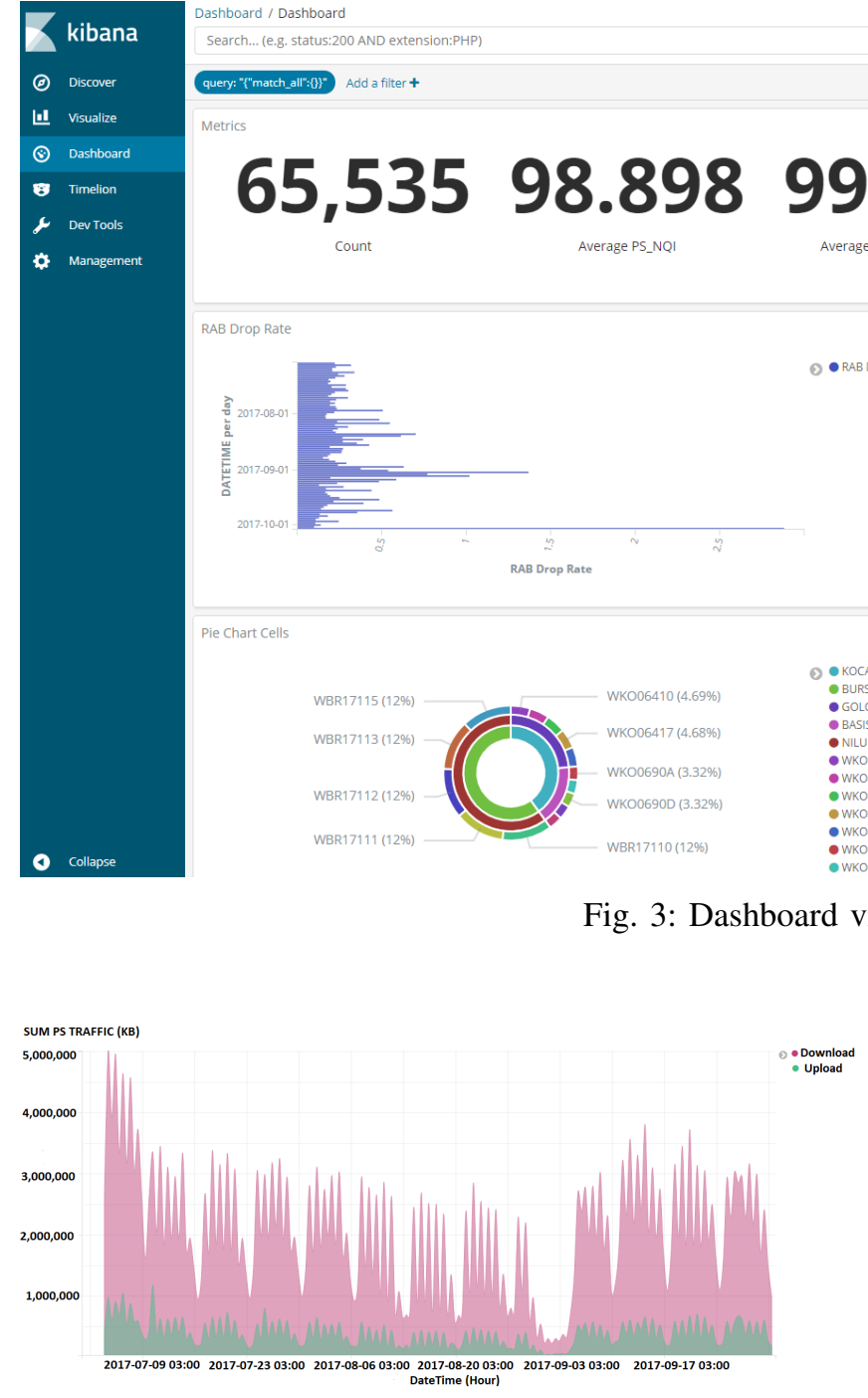

although not as dense as working hours, low-to medium traffic is generated during weekdays.

Fig. 7 shows the UL traffic heatmap median traffic distribution over day-hour of two selected distinctive cells WBR 17133 and WBR 17131. Fig. 7(a) shows that a high traffic occurrence for the cell WBR 17133 on Thursday afternoons. Similarly, Fig. 7(b) shows that on Mondays between 10am to $3 \mathrm{pm}$, high traffic activity have occurred on average.

To observe the behavior of cells over the observed over

Fig. 4: Traffic Distribution over Time (UL/DL).

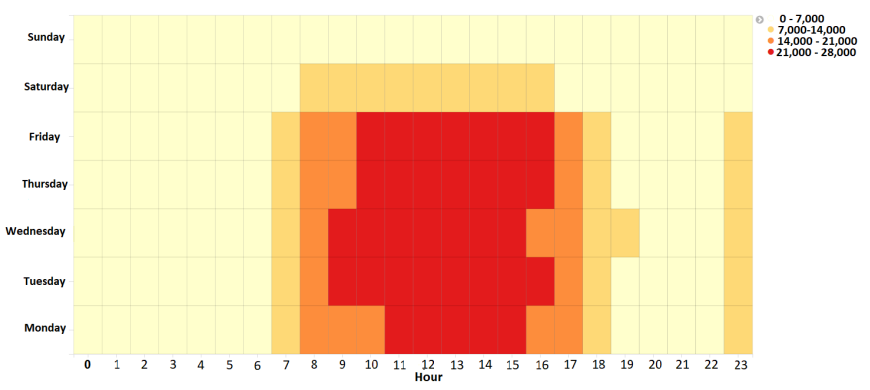

Fig. 5: Factory site cell UL Weekday vs.s hour heatmap. 3 months duration, we also plot the heatmap of the UL traffic (similar behaviors are also observed in DL traffic) over the observed 24 hours and 7 days interval. Every data inside each weekday- hour time zone is the median value of the total UL traffic over the observed duration. Different characteristics of the cell traffic are observed in cells of main building and production building. Fig. 6 shows two of the selected characteristic of distinctive cells over the observed 24 hours and 7 days. Similar characteristics to Fig. 6(a) are also observed in most of the other cells. Fig. 6(b) is another unique characteristics observed on cell WBR17118 where high traffic is observed on Tuesdays between $11 \mathrm{am}$ to $4 \mathrm{pm}$.

Fig. 8 shows the factory cell median values of each month-hour time zone UL heatmap over the observed period. 

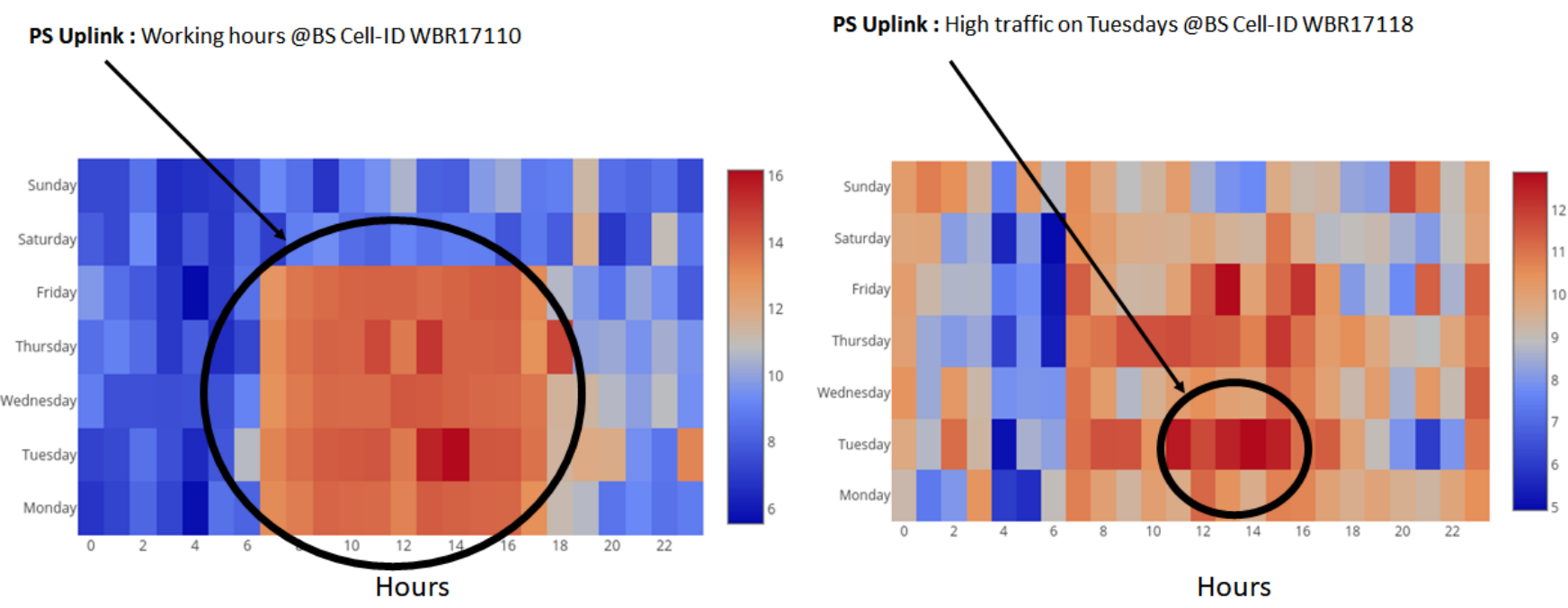

Fig. 6: UL traffic heatmap of two cells in main building (a) WBR 17110 (b) WBR 17118
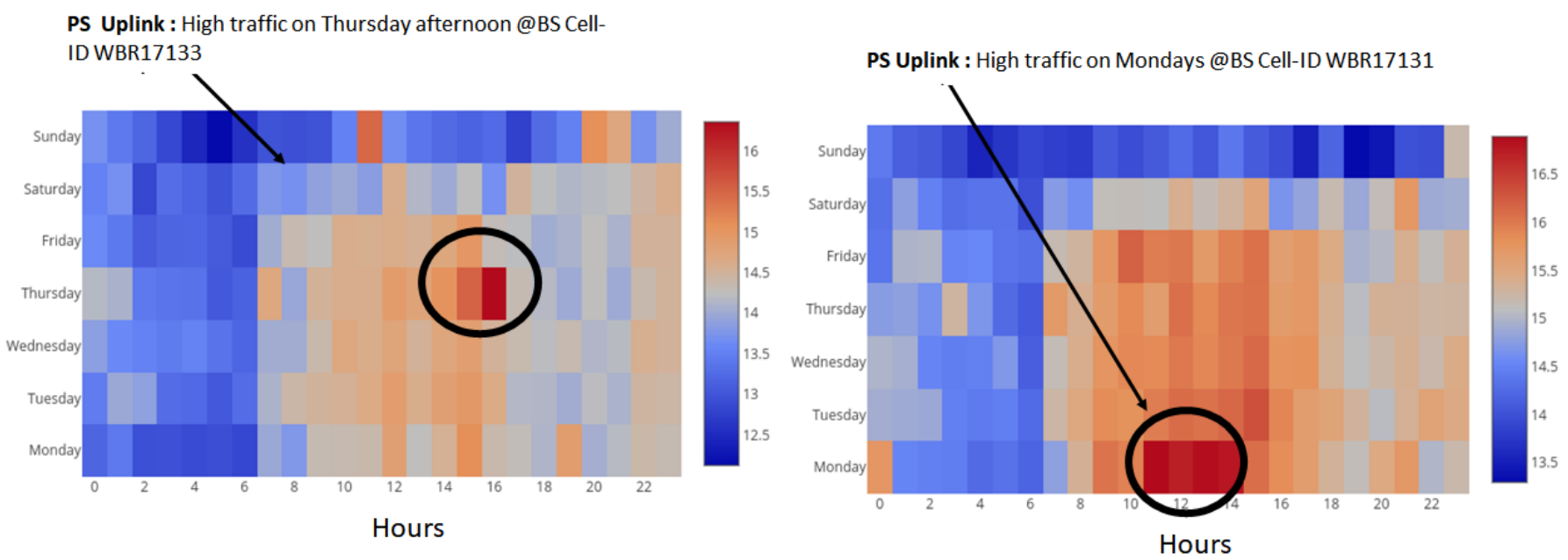

Fig. 7: UL traffic heatmap of two cells in production building (a) WBR 17133 (b) WBR 17131

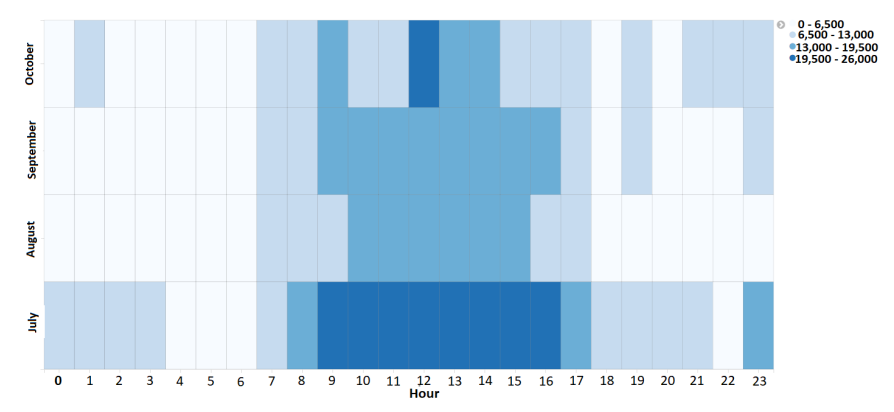

Fig. 8: Factory site cell UL month v.s. hour heatmap

During the observed period of 91 days, we can observe that traffic activity on July are much larger than other months. Interestingly, for the month of July, there exists traffic activity between $11 \mathrm{pm}$ to $3 \mathrm{am}$.

Fig. 9 shows the auto-correlation of the total traffic over the observed duration. We can observe from this figure that there exist high correlation between $24 \mathrm{~h}$ intervals and low correlation between 12 hour intervals. This signifies that the total traffic is following a cyclic daily pattern.

Fig. 10 shows the Spearman's correlation coefficient heatmap of the features. In general, the correlations between the Downlink (DL)/UL traffic and other features in the dataset are not quite highly correlated (negative or positive). NQI metric has high anti-correlation with $R A B_{D R}$ with 0.93 and medium anti-correlation with total traffic with -0.6 . On the other hand, both $R A B_{S R}$ and $R R C_{S R}$ have positive correlation with NQI. The relation between Ret and DL/UL traffic is negative with -0.57 and -0.59 respectively. $R A B_{D R}$ is also linked to DL/UL traffic with positive correlation of 0.57 and 0.59 respectively. The positive correlation between $R A B_{D R}$ and DL/UL traffic signifies that as the total amount of traffic increases so does the $R A B_{D R}$. In case of $A c c$, we can see a low level anti-correlation link between total traffic and 
$R A B_{D R}$ with -0.31 and -0.38 respectively. Small positive correlation (0.27) is visible between $R A B_{S R}$ and $R R C_{S R}$. The correlation between UL and DL traffic is high with 0.95. There is also postive correlation between Ret and Acc with a value of 0.38 .

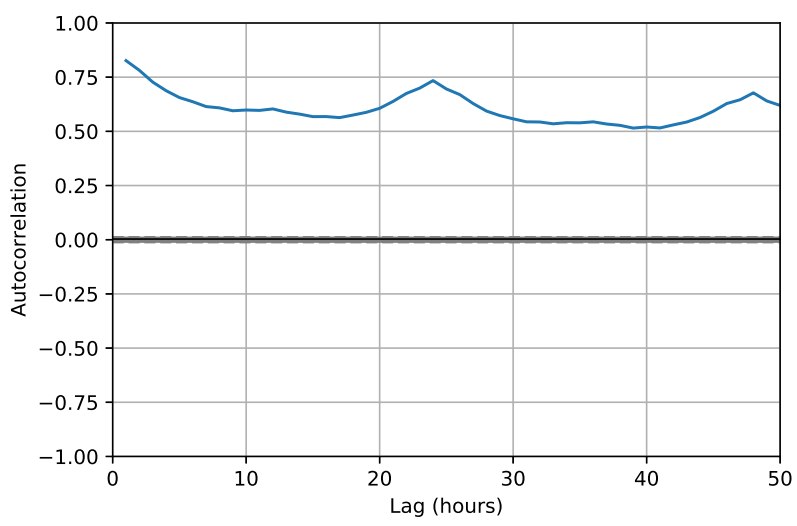

Fig. 9: Auto-correlation function of the total traffic

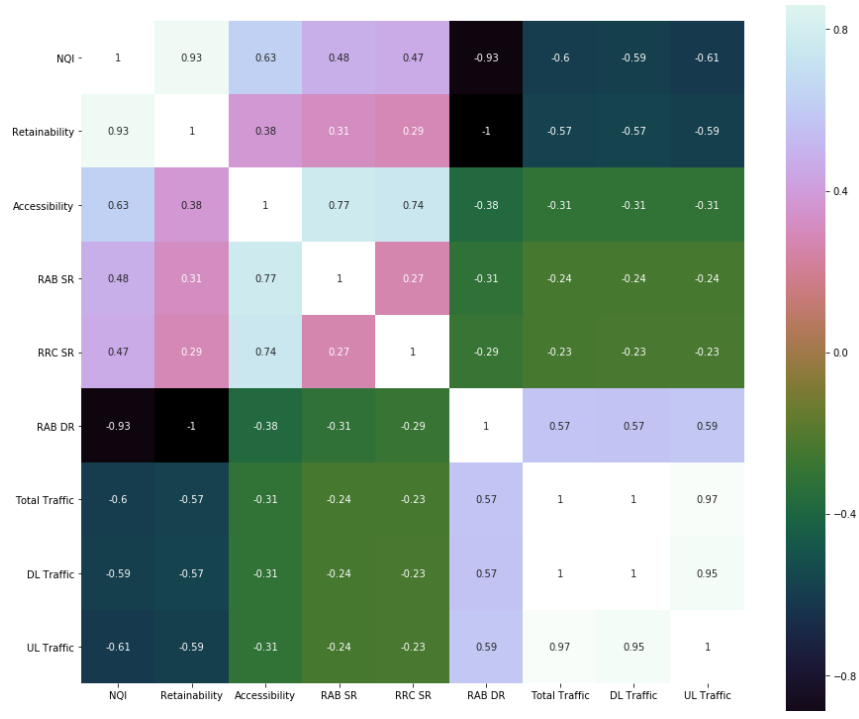

Fig. 10: Correlation heatmap.

\section{CONCLUSIONS AND FUTURE WORK}

Developing a data analytics and visualization platform that can exploit the real-dataset of FoF is important to provide medium to long-term feedback and take appropriate actions in a FoF environment. For this reason, integrating a cuttingedge data analytics platform in a smart FoF environment can ensure to quickly adapt and change to dynamic conditions. In this paper, we have followed an exploratory factor analysis approach to analyze the behaviour of end-users inside the factory environment of one of the automobile factories in Turkey. Our results find some important patterns that may benefit to MNOs as well as factory owners in terms of capacity planning and real-time decision making. As a future work, we are investigating different architectures and techniques to apply machine learning approaches for cellular network traffic analysis of factory environment in real-time.

\section{ACKNOWLEDGEMENT}

This work was partially funded by $\mathrm{H} 2020$ Clear5G project, Spanish MINECO grant TEC2017-88373-R (5G-REFINE) and by Generalitat de Catalunya grant 2017 SGR 1195.

\section{REFERENCES}

[1] S. Mumtaz, A. Alsohaily, Z. Pang, A. Rayes, K. F. Tsang, and J. Rodriguez, "Massive internet of things for industrial applications: Addressing wireless IIoT connectivity challenges and ecosystem fragmentation," IEEE Industrial Electronics Magazine, vol. 11, no. 1, pp. 28-33, 2017.

[2] N. Narkhede, G. Shapira, and T. Palino, Kafka: The Definitive Guide: Real-time Data and Stream Processing at Scale. " O'Reilly Media, Inc.", 2017.

[3] M. Kleppmann, Designing data-intensive applications: The big ideas behind reliable, scalable, and maintainable systems. " $\mathrm{O}$ 'Reilly Media, Inc.", 2017.

[4] S. Landset, T. M. Khoshgoftaar, A. N. Richter, and T. Hasanin, "A survey of open source tools for machine learning with big data in the hadoop ecosystem," Journal of Big Data, vol. 2, no. 1, p. 24, 2015.

[5] A. Oussous, F.-Z. Benjelloun, A. A. Lahcen, and S. Belfkih, "Big data technologies: A survey," Journal of King Saud University-Computer and Information Sciences, vol. 30, no. 4, pp. 431-448, 2018.

[6] E. Zeydan, U. Yabas, S. Sözüer, and Ç. Ö. Etemoğlu, "Streaming alarm data analytics for mobile service providers," in Network Operations and Management Symposium (NOMS), 2016 IEEE/IFIP, pp. 1021-1022, IEEE, 2016.

[7] B. Kurt, E. Zeydan, U. Yabas, I. A. Karatepe, G. K. Kurt, and A. T. Cemgil, "A network monitoring system for high speed network traffic," in Sensing, Communication, and Networking (SECON), 2016 13th Annual IEEE International Conference on, pp. 1-3, IEEE, 2016

[8] N. Albayrak and E. Zeydan, "An intelligent data visualization service platform for mobile network operators," in 2018 Network Traffic Measurement and Analysis Conference (TMA), pp. 1-2, IEEE, 2018.

[9] I. O. Yigit, G. Ayhan, E. Zeydan, F. Kalyoncu, and C. O. Etemoglu, "A performance comparison platform of mobile network operators," in Network of the Future (NOF), 2017 8th International Conference on th, pp. 144-146, IEEE, 2017.

[10] R. Sakellariou, J. Buenabad-Chávez, E. Kavakli, I. Spais, and V. Tountopoulos, "High performance computing and industry 4.0: Experiences from the disrupt project," in 2018 International Conference on Embedded Computer Systems: Architectures, Modeling, and Simulation (SAMOS XVIII), 2018

[11] "Open Source Search \& Analytics - Elasticsearch.” https://www.elastic. co/, 2017. [Online; accessed 06-Jan.-2019].

[12] "Logstash: Collect, Parse, Transform Logs — Elastic." https://www. elastic.co/products/logstash, 2017. [Online; accessed 06-Jan.-2019].

[13] "Kibana: Explore, Visualize, Discover Data — Elastic." https://www. elastic.co/products/kibana, 2017. [Online; accessed 06-Jan.-2019]. 